\title{
Low Momentum Particle Detector for the NA61 Experiment at CERN
}

\author{
Krisztina Márton ${ }^{\mathrm{a}}$, Gábor Kiss ${ }^{\mathrm{a}, \mathrm{b}}$, András Lászlóa ${ }^{\mathrm{a}}$, Dezsô Varga \\ ${ }^{a}$ Institute for Particle and Nuclear Physics, MTA Wigner Research Centre for Physics, Budapest, Hungary \\ ${ }^{b}$ Eötvös University, Budapest, Hungary
}

\begin{abstract}
The NA61 Experiment at CERN SPS is a large acceptance hadron spectrometer, aimed to studying of hadronhadron, hadron-nucleus, and nucleus-nucleus interactions in a fixed target environment. The present paper discusses the construction and performance of the Low Momentum Particle Detector (LMPD), a small time projection chamber unit which has been added to the NA61 setup in 2012. The LMPD considerably extends the detector acceptance towards the backward region, surrounding the target in hadron-nucleus interactions. The LMPD features simultaneous range and ionization measurements, which allows for particle identification and momentum measurement in the 0.1 $-0.25 \mathrm{GeV} / c$ momentum range for protons. The possibility of $\mathrm{Z}=1$ particle identification in this range is directly demonstrated.
\end{abstract}

Keywords: CERN NA61, TPC, centrality measurement, gray proton

\section{Introduction}

Over the last four decades of experimental study of hadronic interactions, a large amount of information has been gathered on production of "slow" particles, which are slow in the sense that in a fixed target environment their rapidity in the target frame is less than unity. The term limiting fragmentation [1] has actually been formulated for this region, and scaling properties have been studied for large variety of reactions.

Considering particle production from a target nucleus in a fixed target hadron-nucleus $(\mathrm{h}+\mathrm{A})$ or nucleusnucleus $(A+A)$ interactions with beam energies in the order of a few $\mathrm{GeV}$, a sizeable low-energy component emerges due to the de-excitation of the nucleus: nucleons or smaller nuclei are produced with kinetic energy of the order of the nuclear binding energy. This component is generally referred to as "black", a name which originates from early emulsion studies [2]. There is an other component, which is strongly connected to the fragmentation of nucleons and is attributed to intranuclear cascading. These "gray" particles, mostly nucleons but also pions and light nuclei, carry kinetic energy of $30-400 \mathrm{MeV}$, considerably higher than the nuclear binding energy (for a complete review, see [3]).

Email addresses: marton.krisztina@wigner.mta.hu (Krisztina Márton), kiss.gabor@wigner.mta.hu (Gábor Kiss), Andras.Laszlo@cern.ch (András László),

Dezso.Varga@cern.ch (Dezsô Varga)
Finally, there is an additional component of the slow particles which resembles that observed in $h+p$ or $h+n$ collisions [4], such as particles in the diffractive peak.

The LMPD (Low Momentum Particle Detector), an integral part of the NA61 Experiment [5], aims at differentiating centrality in $\mathrm{p}+\mathrm{A}$ and $\mathrm{A}+\mathrm{A}$ interactions recorded by the CERN experiment NA61, and to clarify quantitatively the details of the relation between event centrality and slow particle production. NA61 has a key advantage having high acceptance, allowing identified produced particles (including strangeness content, central baryons and antibaryons) and slow particles (by LMPD) to be measured by the same apparatus [6].

\subsection{Centrality control in $h+A$}

The production of low momentum particles in high energy hadron-nucleus collisions were studied by many experiments over the last few decades [7]. A key observation was that the number of the slow nucleons, especially in the "black" and "gray" regions emerging from the break-up of the nucleus, gives information about the centrality (the impact parameter) of the $\mathrm{h}+\mathrm{A}$ collision.

$\mathrm{The} \mathrm{h}+\mathrm{A}$ collisions were studied at various energies, with different types of projectiles and targets. It was found that the angular distributions of the low momentum ("gray" and "black") protons are to first order independent of the energy and of the type of the incoming projectile, but they show significant dependence on the 
mass of the target nucleus: they are stronger forwardpeaked for lighter targets. The angular distributions for "gray" protons are forward-peaked, while for the "black" ones show only little asymmetry [2, 8, 9].

Regarding $\mathrm{p}+\mathrm{C}$ interactions, a comprehensive data survey with critical review of compatibility between various measurements has been recently published [10], incorporating relevant new measurements by the NA49 Experiment in $\mathrm{p}+\mathrm{C}$ interactions [11]. This completes earlier discussion of the $\mathrm{p}+\mathrm{C}$ collision system [4], clarifying the momentum regions populated by the different production mechanisms.

The yield of these slow protons in the $\mathrm{h}+\mathrm{A}$ interaction is found to have two sources: the nucleon-nucleon encounters and the processes involving nuclear matter. This latter group of processes have also important role in the production of deuterons, tritons and other light nuclei [12].

It was suggested already in 1976 [13] that by measuring the large composite fragments, one can select the central collisions, and subsequently it was proposed [14] that the number of heavily ionizing particles $N_{h}$ measures the number of struck nucleons inside the target nucleus.

The energy independence of the distribution of these heavy particles supported the hypothesis that $N_{h}$ measures the impact parameter (the centrality) of the $h+A$ collision and it is correlated to the number of nucleonnucleon collisions in the nucleus [15]. With increasing centrality the number of "gray" nucleons increases almost proportionally, whereas the number of "black" particles saturates for central collisions [16].

One of the aims of the LMPD is to understand the transition from "black" to "gray" energy ranges, and to quantify how these regions are related to centrality. Our choice of technology matches this region, with best performance (proton identification and momentum measurement) in the $15-30 \mathrm{MeV}$ kinetic range, well covering the transiton from "black" to "gray". "Gray" protons up to $500 \mathrm{MeV} / \mathrm{c}$ momentum (120 MeV kinetic energy) can be tagged by their high ionization deposit.

\subsection{NA61 environment}

During normal physics data taking in 2012, the LMPD was an integrated part of the NA61 detector system. The position of the LMPD for these periods is indicated in Figure 1 surrounding the target. Also, data has been taken for a considerable time in 2011 in a downstream position, to exploit the available beam time for configurations incompatible with the LMPD (use of hydrogen target). In this case the LMPD was independent from the rest of the NA61 system, with a stand-alone trigger, data acquisition, and target setup.

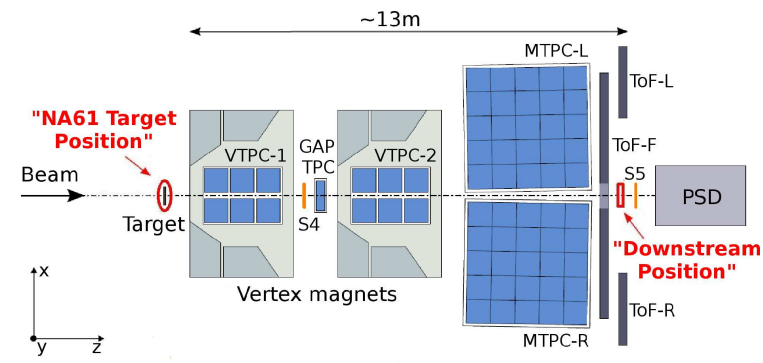

Figure 1: Outline of the NA61/SHINE Experiment. LMPD data taking positions are indicated.

Table 1: Collected data at $158 \mathrm{GeV} / \mathrm{c}$ in "downstream" position (2011, standalone) and in "NA61 target" position (2012, full NA61).

\begin{tabular}{|c|c|c|}
\hline Year & Target & Collected events \\
\hline \multirow{4}{*}{2011} & Pb, $0.5 \mathrm{~mm}$ & $2442 \mathrm{k}$ \\
& $\mathrm{Pb}$ (rotated), $0.5 \mathrm{~mm}$ & $617 \mathrm{k}$ \\
& $\mathrm{C}, 2 \mathrm{~mm}$ & $547 \mathrm{k}$ \\
& $\mathrm{A} 1,1 \mathrm{~mm}$ & $622 \mathrm{k}$ \\
& Target out & $264 \mathrm{k}$ \\
\hline \multirow{4}{*}{2012} & ${ }^{33} \mathrm{Kr}($ calibration) & $1593 \mathrm{k}$ \\
\cline { 2 - 3 } & Pb, 0.5mm & $2140 \mathrm{k}$ \\
& Target out & $274 \mathrm{k}$ \\
\cline { 2 - 3 } & Pb, 1mm & $9206 \mathrm{k}$ \\
& Target out & $927 \mathrm{k}$ \\
\hline
\end{tabular}

This downstream position, behind the MTPCs (see also Figure 1) allowed a flexible change of operational conditions, therefore most of the technical studies were performed here.

The integration of the LMPD unit into the NA61 environment was largely simplified by the fact that the LMPD uses the same front-end electronics as the existing NA61 TPCs. The detector has been included in NA61 data acquisition system and the online monitoring system as well, in a fashion compatible with all the other TPC units.

\section{Detector construction}

\subsection{Principle of operation}

The detector exploits the simultaneous measurements of ionization $(\mathrm{dE} / \mathrm{dx})$ and range, which, due to the different mass, makes a differentiation between particle types. 
The range measurement is rough, typically with a precision of a factor of two; this is however sufficient to specify a narrow momentum bin, since the momentum dependence of range is very steep (approximately proportional to the fourth power of the momentum). The ionization ratio at a given range for any two types of particles is approximately proportional to the square root of the mass ratio: this implies that also the $\mathrm{dE} / \mathrm{dx}$ measurement need not to be very precise for clear identification (between pions and protons the ionization ratio is around 2.6 for a given range). For low momentum particles of interest the ionization is high (about 5-20 times the minimum), allowing sampling in a gas gap of a few $\mathrm{cm}$. In addition, the per event multiplicity of these particles is rather low, up to a few tens with an approximately spherical distribution. These considerations led to a rather compact detector outline, where position sensitive detection layers are interspersed with absorber layers. The thin detector walls imply operation at atmospheric pressure, specifically, about $0.2-0.5$ mbar above ambient pressure.

The actual design was guided by a simulation based on the Photon Absorption Ionization (PAI) [17] model. The comparison between the simulation and the measurements are discussed in detail in Section 5.3.

\subsection{Detector outline}

The Low Momentum Particle Detector is a small time projection chamber with absorber layers in the gas volume. The detector outline following the principles discussed in Section 2.1 is shown in Figure2 The absorber layers define intervals in the range of the particles and they also act as an inner field cage. The vertical electric field in the LMPD guides the produced ionization electrons drifting towards the top of the detector, where they are read out by a multi wire proportional chamber.

LMPD has two independent parts, the "Jura"- and the "Saleve"-sides, see Figure 3 Figure 4 shows the absorber layers and field cage of "Jura-side". The absorbers are glass-epoxy (G10) sheets with $2 \mathrm{~mm}$ wide horizontal $\mathrm{Cu}$ strips. The outer field cage is a $60 \mu \mathrm{m}$ kapton foil printed with $5 \mu \mathrm{m} \mathrm{Cu}$ strips. The readout MWPC has approximately radial pad structure. There are 10 pad rows, the absorbers are after every second pad row, defining 5 detection layers. (More details about the readout chamber are in Section 2.5)

In 2010 a prototype of LMPD was also built. This "2010 Proto" unit was found to be useful as a multiplicity monitor in the downstream setup, in combination with the final detector.

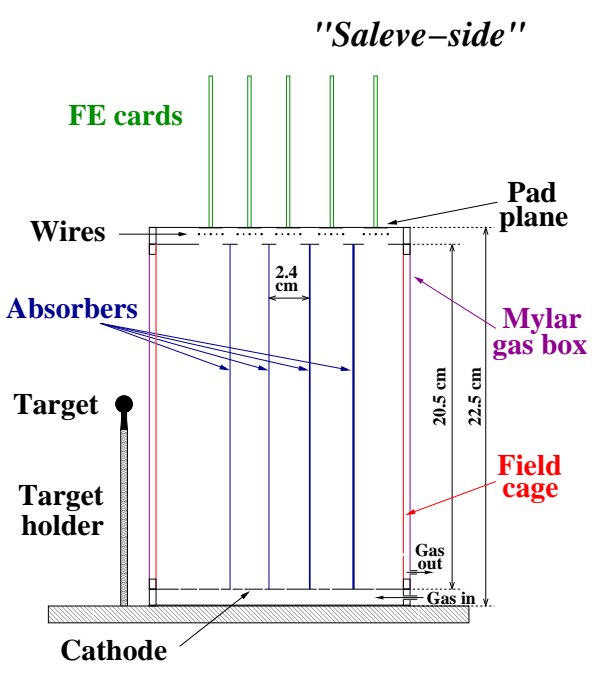

Figure 2: Detector outline (one half) from the beam direction.

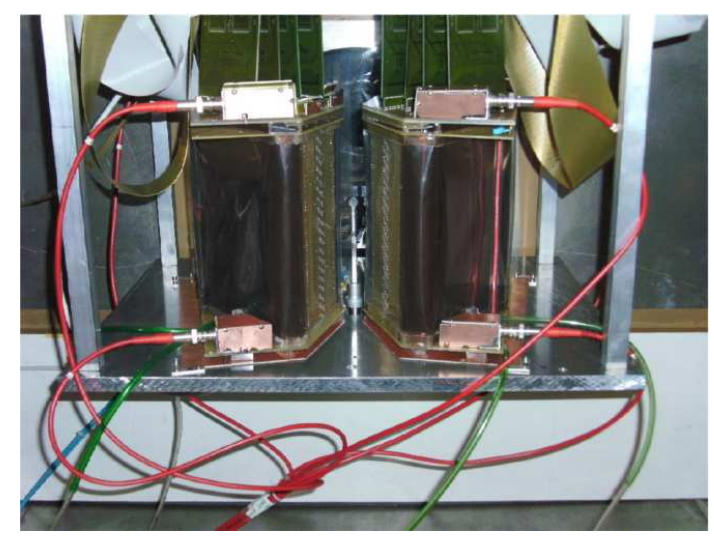

Figure 3: Complete system (LMPD+target) in "NA61 target" position.

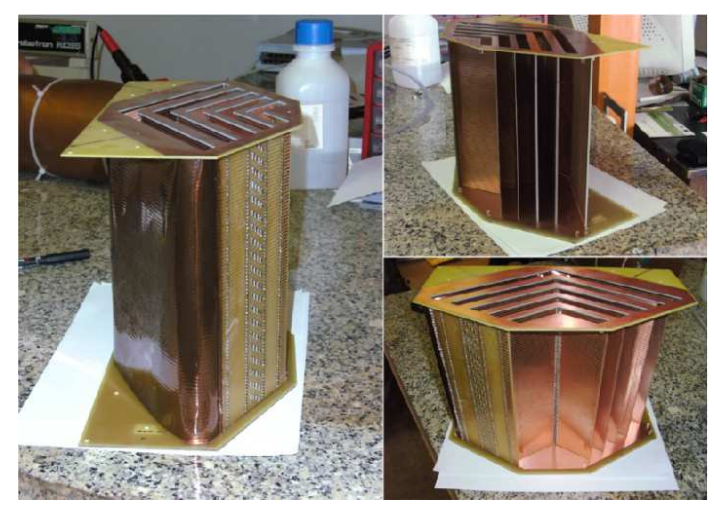

Figure 4: LMPD: absorber layers and field cage of "Jura-side". 


\subsection{Gas system}

LMPD has double walls, similarly to the other TPC chambers of the NA61 detector. The inner wall is a $60 \mu \mathrm{m}$ kapton layer, the outer one is a $40 \mu \mathrm{m}$ thick mylar foil. Mixture of $85 \% \mathrm{Ar}+15 \% \mathrm{CO}_{2}$ has been used as filling gas.

The gas enters from the bottom part of the chamber through 30 holes drilled in the cathode plane, with $1 \mathrm{~mm}$ diameter each, in order to evenly distribute the fresh gas between the absorber layers. The used gas is then guided to fill the layer between the kapton and the mylar foil before being vented from the chamber. This solution allows one to exploit the quality improvement achieved by the double wall structure, without the need of an additional gas circulation path.

\subsection{Absorber structure}

The absorber structure of LMPD is shown in Figure 4. There are 4 absorber layers in both Jura- and Saleve-sides, placed after every second pad row. The absorbers are made from glass-epoxy. Their thicknesses are $0.5,1.0,2.0$, and $2.5 \mathrm{~mm}$, however the effective thickness depends on the angle of incidence. The detector wall acts as an absorber, and hence defines the minimum detectable particle energy.

As a general overview, Table 2 gives the basic properties of the absorbers, as well as the momentum cutoff for protons which are able to pass through the given absorber layer. These latter quantities, especially the ionization, have a complicated dependence on the particle and detector geometry as well as the energy distribution, therefore will be subject of a detailed analysis. The key message of the present paper is to demonstrate the possibility of a clean measurement for these approximate kinematic ranges.

\subsection{Readout $M W P C$}

The readout chamber is a MWPC which is placed on the top of the chamber, with segmented cathode (pads) on ground potential. The close to radial pad-structure is shown in Figure 5. The pads are organized in 10 rows perpendicularly to the typical track direction, the number and size of pads increase towards the outer pad rows.

In the readout chamber there are two kind of wires, the sense (anode) wires with $21 \mu \mathrm{m}$ thickness and the field wires (100 $\mu \mathrm{m}$ thick). The wires are to first order compatible with the radial structure of pads with the help of a wire-holder in the middle of the pad plane, which bends the wires on a short section and therefore reduces the overall dead zone. The wire-holder in the middle divides the pad-structure to two symmetric parts

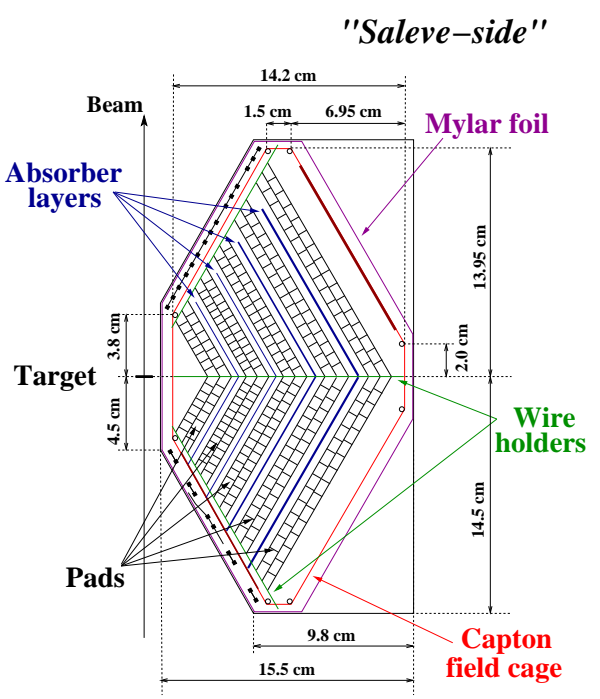

Figure 5: Pad-structure of the "Saleve-side" detector.

("wedges", see Figure 5]. These wedges are handled independently during the analysis.

The pads are $6 \mathrm{~mm}$ long, that is, the track segment between each absorber pair is measured on a $12 \mathrm{~mm}$ segment. The distance between sense wires are correspondingly $6 \mathrm{~mm}$. The distance between the wire plane and the pad plane is $4 \mathrm{~mm}$.

The signal formation is based on the same principle as for the larger TPC-s of the NA61 detector [6, 18], with avalanche formation on the sense wires, and capacitive signal coupling to the pads.

Since the LMPD detects highly ionizing slow particles, the optimal gas multiplication gain is below the typical TPC gains designed for minimum ionization. This implies that even gating grid is not necessary, which would otherwise reduce ion backflow to the TPC sensitive volume. The total current measured on the sense wire high voltage supply line was typically $20 \mathrm{nA}$ at full beam rate, that is, around $0.07 \mathrm{nA} / \mathrm{cm}$ current density, which justified this approach.

The typical proton momentum is lower for those tracks which stop early, and higher for those which run along all the detection layers. In order to optimize the electronics dynamic range, a gradually increasing gas multiplication gain has been applied towards outer pad rows to follow the decreasing ionization due to increasing momentum. The practical realization relied on a resistor chain (see Figure 6), with a constant voltage drop between each absorber layers for the sense wires. 
Table 2: Absorber thicknesses, approximate momentum ranges and ionization (in $1.2 \mathrm{~cm} \mathrm{Ar)} \mathrm{for} \mathrm{perpendicular} \mathrm{incidence.}$

\begin{tabular}{|c|c|c|c|c|c|}
\hline $\begin{array}{c}\text { Absorber } \\
\text { number }\end{array}$ & $\begin{array}{c}\text { Thickness } \\
(\mathrm{mm})\end{array}$ & $\begin{array}{c}\text { Thickness } \\
\left(\mathrm{g} / \mathrm{cm}^{2}\right)\end{array}$ & $\begin{array}{c}\text { Cumulative } \\
\text { thickness } \\
\left(\mathrm{g} / \mathrm{cm}^{2}\right)\end{array}$ & $\begin{array}{c}\text { Momentum } \\
\text { cutoff }(\mathrm{MeV})\end{array}$ & $\begin{array}{c}\text { Most probable } \\
\text { ionization } \\
(\mathrm{keV})\end{array}$ \\
\hline Detector wall & 0.1 & 0.018 & 0.018 & 110 & - \\
\hline 1 & 0.5 & 0.106 & 0.124 & 127 & 100 \\
\hline 2 & 1.0 & 0.213 & 0.337 & 171 & 63 \\
\hline 3 & 2.0 & 0.425 & 0.762 & 216 & 38 \\
\hline 4 & 2.5 & 0.532 & 1.294 & 250 & 26 \\
\hline
\end{tabular}

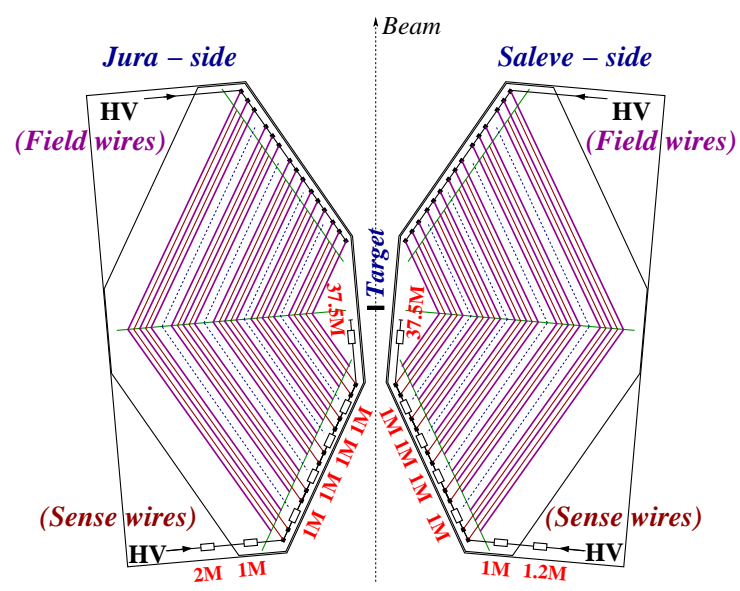

Figure 6: Top view of the LMPD: mechanical and high voltage support for the wires, with the resistor chain on the sense wires indicated.

\subsection{Simulation of electron drift}

In order to find the appropriate voltage settings, electrostatic simulations have been performed with Garfield [19]. As the geometrical properties of LMPD require thin wires and relatively large planes as well, the nearly exact Boundary Element Method solver [20] was used to calculate the electric field (Garfield is interfaced with the neBEM program).

Figure 7 shows the equipotential lines in the vicinity of the wire plane, as well as part of the field cage. The absorber walls are vertical (y coordinate), and the wires are perpendicular to the plane of the Figure. On the right side of Figure 7 several calculated electron drift lines are shown, starting from $y=2.2 \mathrm{~cm}$ position. This demonstrates that with these voltage settings the majority of electrons are collected by the anode wires. Note that further from the amplification cell, the field structure is completely defined by the field cage, that is, the voltage
Table 3: High voltage settings during the physics run.

\begin{tabular}{|c|c|c|}
\hline & Saleve-side & Jura-side \\
\hline Cathode HV & $-4000 \mathrm{~V}$ & $-4000 \mathrm{~V}$ \\
\hline Field Wire HV & $-400 \mathrm{~V}$ & $-400 \mathrm{~V}$ \\
\hline Sense Wire HV & $1150 \mathrm{~V}$ & $1150 \mathrm{~V}$ \\
\hline $\begin{array}{c}\text { Pad row 1,2 - } \\
\text { SW }\end{array}$ & $987 \mathrm{~V}$ & $969 \mathrm{~V}$ \\
\hline $\begin{array}{c}\text { Pad row 3,4 - } \\
\text { SW }\end{array}$ & $1013 \mathrm{~V}$ & $995 \mathrm{~V}$ \\
\hline $\begin{array}{c}\text { Pad row 5,6 - } \\
\text { SW }\end{array}$ & $1040 \mathrm{~V}$ & $1021 \mathrm{~V}$ \\
\hline $\begin{array}{c}\text { Pad row 7,8 - } \\
\text { SW }\end{array}$ & $1066 \mathrm{~V}$ & $1047 \mathrm{~V}$ \\
\hline $\begin{array}{c}\text { Pad row 9,10 - } \\
\text { SW }\end{array}$ & $1092 \mathrm{~V}$ & $1073 \mathrm{~V}$ \\
\hline
\end{tabular}

settings have no effect on the collection efficiency.

The simulations confirmed the approach in which a single wire layer was installed, simplifying the construction step. This implied however, that the field wires are set on a considerable negative voltage, $-400 \mathrm{~V}$ for all field wire voltages. Such setting reduced the gain dependence on cathode flatness [21], and thus improved gain uniformity.

\subsection{Read-out and electronics}

Electronic signals from each of the individual cathode segments (pads) in the readout MWPC are recorded by the same front-end (FE) cards as used for the NA61 tracking TPCs [22, 23]. Each of these FE cards can store analog time trace of 32 TPC pads, with time sampling 

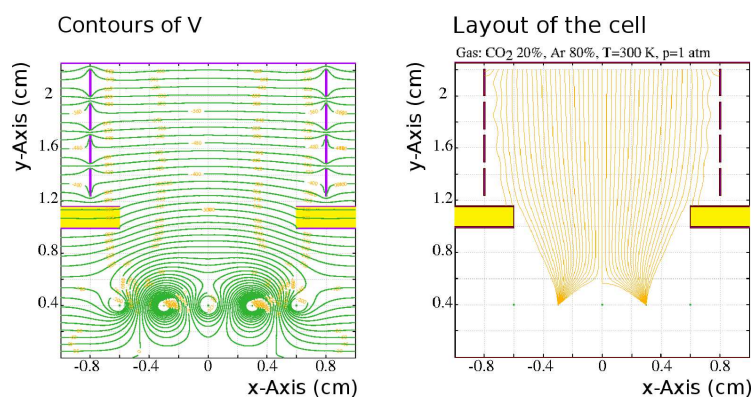

Figure 7: Left panel: equipotential lines in the LMPD amplification cell (two pad rows out of ten). Right panel:drift lines of electrons.

in 256 elements of $200 \mathrm{~ns}$ spacing, allowing total drift time of $51.2 \mu \mathrm{s}$. After sampling, the FE cards digitize the signals in a serial way using an on-card Wilkinson ADC. LMPD uses 18 such FE cards.

The steering logic for the FE readout process is hosted on the readout mother boards (MB). The 9 bit pad charge ADCs from the FE cards are pedestal subtracted, truncated to 8 bit, noise suppressed and zero compressed by the MB before serializing them to an LVDS connection line towards a concentrator box (CB). These further serialize the data to a DDL optical connection line [24, 25] towards the Central DAQ computer of the NA61 experiment. One MB can host up to 24 pieces of FE cards, thus only one is used for the LMPD (including all subunits).

The detected signal shapes, timing and the noise performance was compatible with that experienced at the other NA61 TPCs. The electronics control and maintenance (including regular pedestal measurements, monitoring of power supply and data stream) was integrated into the NA61 framework.

Figure 8 shows event display of ADCs of a typical raw event, available for on-line performance checks during the measurement.

\section{Target and trigger system}

\subsection{Trigger counters}

The detector by design operates with a target which is as thin and as narrow as possible. To reduce background, various trigger counters were arranged in an optimum way.

During the 2011 data taking in "downstream position" (Figure 1), the signal from three plastic scintillators were combined, in coincidence with the incoming beam particle (defined by the NA61 beam trigger). The last two scintillators were close to the target. The one at

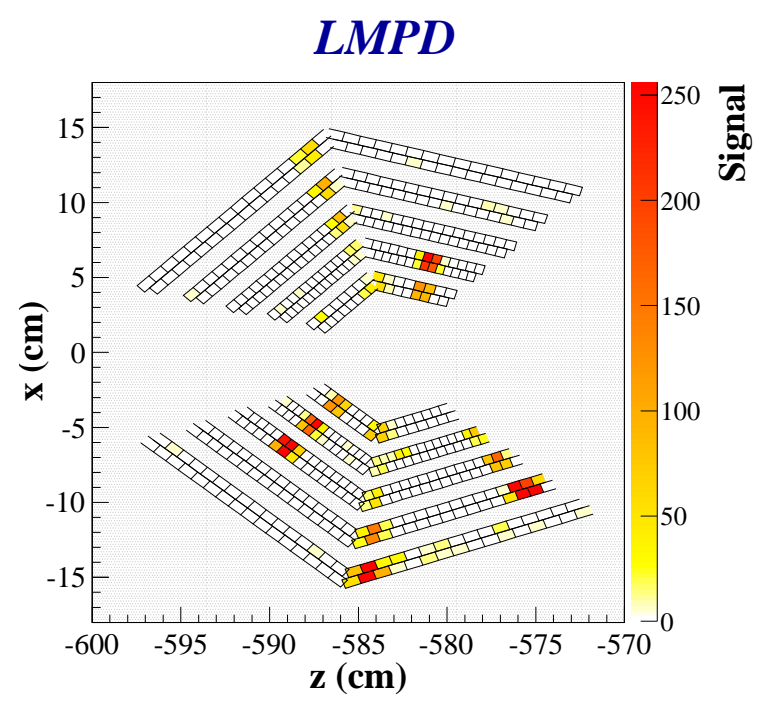

Figure 8: Typical raw event of LMPD (top view).

$40 \mathrm{~cm}$ was a $2 \mathrm{~cm}$ wide, $5 \mathrm{~mm}$ thick disc, whereas the one at $30 \mathrm{~cm}$ was $5 \mathrm{~cm}$ by $5 \mathrm{~cm}$ with $2 \mathrm{~mm}$ thickness. The elimination of beam halo was achieved by an additional counter (LMPD-V0) in anti-coincidence, right in front of the target. It was $6 \mathrm{~cm}$ circular scintillator, with $1 \mathrm{~cm}$ thickness, and with a $5 \mathrm{~mm}$ diameter hole in the middle. The material budget for LMPD-V0 in the hole was minimized to efficiently reduce background. The outline is shown in Figure 9. The interactions taking place in the target were captured by an additional $2.5 \mathrm{~cm}$ by $3 \mathrm{~cm}, 2 \mathrm{~cm}$ thick scintillator, $4.5 \mathrm{~m}$ behind the target (LMPD-S3). The geometrical alignment of the setup was very critical in reaching high signal to background ratio, and was carefully verified by beam scans.

In the "target position" (Figure 1) the trigger definitions of NA61, similar to the former NA49 experiment, were used [18]. The beam was defined by the coincidence of two scintillators (S1 and S2 on Figure 9) in anticoincidence with two veto counters (V0 and V1 in Figure 9. To get identified proton beam, a CEDAR Ring Cerenkov Counter was used.

\subsection{Target system}

During the data collecting periods, targets of different atomic number (A) and thicknesses were used. In order to estimate the background from non-target interactions, the target was removed regularly ("target out" measurements). The switch between target in and out positions was performed with a remotely controlled pneumatic moving mechanism, which eliminated the necessity of 


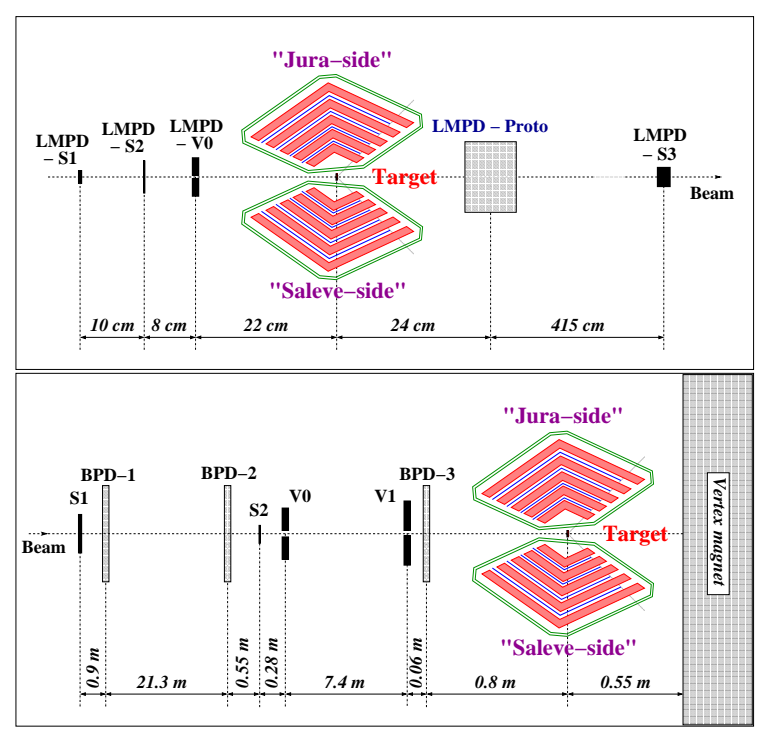

Figure 9: Outline of the complete system in downstream (upper panel) and in target position (lower panel; not to scale, note distances indicated).

entering the experimental area, and hence improving data taking efficiency. During the physics run in 2012 a thin Tedlar foil He pipe was installed around the target to reduce the background.

In the downstream position the beam quality was less controlled compared to the case of the NA61 nominal target region. Due to the small diameter of the target, precise alignment of the beam spot, the target and the trigger counters (LMPD-V0 and LMPD-S3) was mandatory. Besides optical alignment, we have opted for a direct alignment cross-check based on actual particle data. To this end, the 2010 Prototype was used as a monitor for incoming beam particle positions for some of the data taking time.

\section{Krypton calibration}

For the read-out of our detector $2 \times 9$ FE cards are used, each of them has 2 amplifier chips with $16+16$ channels. Since the amplification of the chips can be different, the gain may vary pad by pad. For the relative gain calibration of the pads, random trigger events with ${ }^{83} \mathrm{Kr}$ source were collected. This method came from the ALEPH experiment and it was used also in DELPHI and in NA49/NA61 experiments [18].

${ }^{83} \mathrm{Kr}$ is an isotope which is produced by electron capture from ${ }^{83} \mathrm{Rb}$. The ground state of ${ }^{83} \mathrm{Kr}$ is not populated directly, the decay chain results a rich structure of electron energies in the range of $9-42 \mathrm{keV}$. During the calibration data taking, a foil doped with ${ }^{83} \mathrm{Rb}$ was placed into the existing gas system via a bypass line. The gaseous ${ }^{83} \mathrm{Kr}$ isotope could be easily distributed in the chambers, whereas due to the short lifetime of ${ }^{83} \mathrm{Kr}$, no disposal of radioactive gas was necessary and the chambers could be operated normally after few halflives [26].

For the analysis of the Kr data collected with LMPD, a "3D cluster finder" was used which processed in combination the pair of pad rows between two adjacent absorbers. This approach was useful in reducing charge leakage between the closeby pad rows. The calibration was made iteratively, the linearity of the detector response was checked. The Kr spectrum from the NA49 experiment and measured with the LMPD (on a single pad, reconstructed with the 3D cluster finder) is shown in Figure 10.
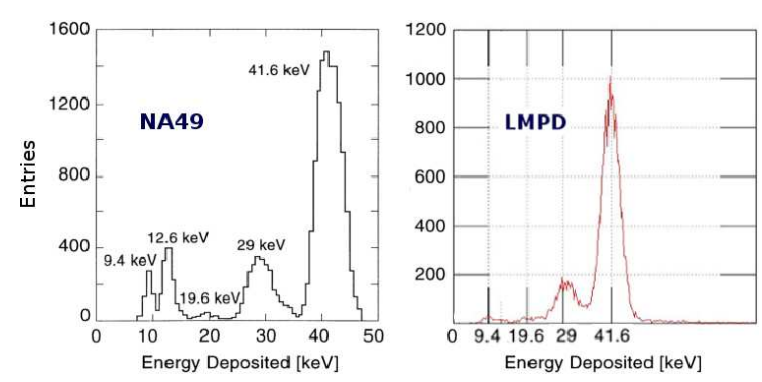

Figure 10: Krypton spectra from NA49 (left, [18]) and measured with LMPD reconstructed by a 3D cluster finder (right).

Figure 11 shows the $\mathrm{Kr}$ spectrum for all pads in LMPD made by the 2D cluster finder (the same as used for the analysis of physics data, optimized for tracking) before and after the calibration. After the calibration the structure of the $\mathrm{Kr}$ spectrum is visible, the apparent background at low values is a result of charge leakage between adjacent pads and pad rows. The position of the $41.6 \mathrm{keV}$ peak on each pads is shown on Figure 12. The distribution on the right panel is fitted with a Gaussian, resulting in sigma/mean value of $3.3 \%$. This figure demonstrates the relevance and necessity of the $\mathrm{Kr}$ calibration, resulting in a highly reliable equalization of the gains.

\section{Performance and pilot data taking results}

\subsection{Event reconstruction and performance}

The first step of event reconstruction is the finding of clustered high ADC hits on the pad row - time sampling detection planes, which correspond to the ionization signals left on a given detection plane (pad row) 


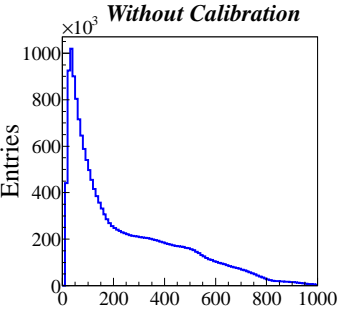

$\mathrm{Kr}$ cluster charge (ADC units)

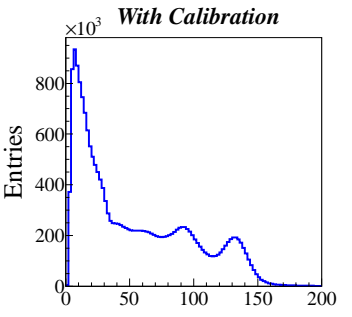

$\mathrm{Kr}$ cluster charge (ADC units)
Figure 11: Kr spectra on all pads (before and after calibration), reconstructed with the $2 \mathrm{D}$ tracking cluster finder.
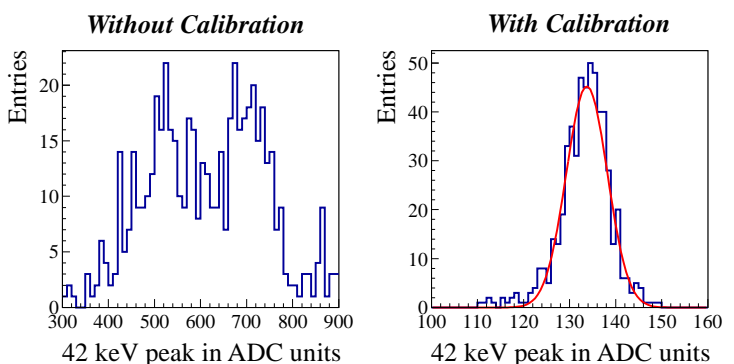

Figure 12: Position of the dominant $41.6 \mathrm{keV}$ peak of the $\mathrm{Kr}$ spectra in ADC units before and after the calibration. The relative fluctuation is $3.3 \%$ after calibration.

by the track of the charged particles. For this task, we applied a simple closest neighbor search algorithm: if any of the charge ADC values on the pad-time plane was at least $C_{\text {high }}=9$ ADC, neighbor search was initiated around it. In case a neighbor had at least $C_{\text {low }}=6$ ADC charge amplitude, it was considered to belong to the same cluster of hits, and its neighbors were also searched for charge above the $C_{\text {low }}$ threshold recursively. The value of $C_{\text {low }}$ and $C_{\text {high }}$ were a result of optimization, motivated by the typical electronic noise level, which was order of $\sigma \approx 3$ ADC. This means that neighbor search was initiated with at least $3 \sigma$ amplitude level and was continued recursively with at least $2 \sigma$ amplitude level, whereas maxADC of a typical true signal cluster was order of $10 \sigma$ or higher. Therefore, these settings are expected to guarantee effective noise rejection along with good cluster finding efficiency. Indeed, a study of cluster charge distribution with varying $C_{\text {high }}, C_{\text {low }}$ values showed that the contribution of true signal clusters are not effected by our particular choice of these cuts and confirms our expectation. After clusterization of hits, the cluster properties are constructed by weighted averaging with the charge amplitude. In such a way for each cluster the centroid and the elongation parameters on the given detection plane are calculated. Elongation parameters are also used for electronic noise rejection: due to the construction of the FE electronics a typical noise cluster is only one timeslice in time direction but extended in the direction of pads, which largely differs from clusters of track signals being approximately circular in shape. Our particular way of cluster centroid calculation is also commonly referred to as center of gravity (COG) method. Studies show [27] that centroid estimation algorithms with smaller bias and better resolution also exist. However, in our case the simple COG approach was applied as the signal clusters consisted of large number of hits with high amplitude, the position resolution was dominated by the multiple scattering in the absorber layers, furthermore, precision tracking was not necessary for our purpose as only multiplicity counting was performed in a relatively low population detection environment.

The second step of the reconstruction is finding of particle trajectories, which are straight tracks of clusters in the detection volume. For this task, first a simple combinatorial track finder was applied. Clusters starting from the target were gathered into track candidates combinatorically, with first considering the longer candidates with less number of unregistered intermediate clusters. These candidates were fitted with straight line hypothesis assuming the same and arbitrary cluster position uncertainty everywhere to construct the $\chi^{2}$ expression to be minimized. The $\chi^{2}$ distribution of the true and false candidates showed a very good separation, and this separation cut was used to define accepted candidates. The clusters of the accepted track candidates were not considered for the generation of further candidates. The distribution of the deviation of the cluster centroids from the fitted tracks was used to determine the position resolution of the centroid determination method as shown in Figure 13. the position resolution in the pad direction was seen to be order of $0.5 \mathrm{~mm}$, while $0.7 \mathrm{~mm}$ in the drift direction. The measured cluster centroid resolution values were used to construct a statistically accurate $\chi^{2}$ expression for track finding and fitting which was then used in the reconstruction of the total recorded data.

The combinatorial track finding, however, proved to be very costly in computational time in case of events with larger number of clusters, furthermore the relative high probability of cluster responses below detection threshold posed a complication: one needs to find track patterns with possibly missing intermediate measured points while minimizing the inclusion of noise clusters. This motivated the development of a track find- 


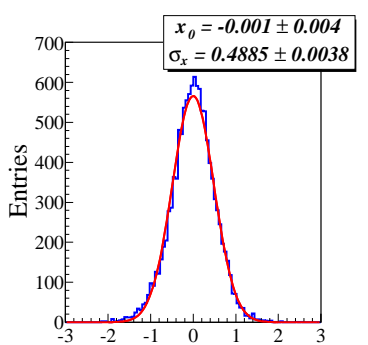

Horizontal resolution $(\mathrm{mm})$

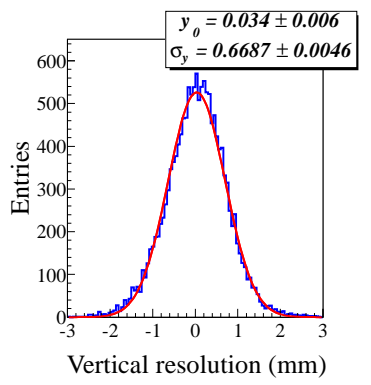

Figure 13: Determination of position resolution of cluster centroids via their deviation from fitted tracks (histogram: data, solid line: Gaussian fit).

ing method whose cost was not increasing factorially with the number of tracks in the event. Our choice fell to Hough transformation [28] combined with maximum likelihood principle.

The basic idea of Hough transform is that the position of a cluster centroid determines a hyper-plane in the four dimensional vector space of straight track parameters through the identities $X=M_{X}+Z N_{X}$ and $Y=M_{Y}+Z N_{Y}$, where $Z$ is our affine parameter along our track, $M_{X}, M_{Y}, N_{X}, N_{Y}$ are our track parameters, while $X, Y$ are the cluster centroid coordinates at a $Z=$ const plane through which the track is required to pass. The intersection of such hyper-planes determines the straight line tracks. In order to capture the described hyper-planes the track parameter space is uniformly binned in each direction, the parameters $N_{X}, N_{Y}$ are scanned as free parameters of the $M_{X}=X-Z N_{X}$, $M_{Y}=Y-Z N_{Y}$ plane, and the corresponding intersected $M_{X}, M_{Y}$ bins are marked as possible track parameters.

According to the Hough method, the parameter space bins where lots of hyper-planes pass through are considered as track candidates. This method is known to be very sensitive to careful choice of parameter space bin size, as with too large bins clusters belonging to different tracks may be accidentally merged to a single track, while with too small bins only very few planes of the same track will intersect in the very same point due to finite resolution of cluster centroid positions. Motivated by this, we implemented an improved version of Hough transformation.

In the improved version, for each cluster centroid the position resolution obtained with the described combinatorial method is also used. For each such position measurement $X \pm \sigma X, Y \pm \sigma Y$ at $Z=$ const the $\pm 3 \sigma$ band $\delta M_{X}=3 \sigma X+|Z| \delta N_{X}, \delta M_{Y}=3 \sigma Y+|Z| \delta N_{Y}$ around the nominal Hough plane is considered. For each intersection bin of these $3 \sigma$ plane bands the statistical $\chi^{2}$ is calculated using the error propagation formula $\sigma^{2} M_{X}=\sigma^{2} X+\frac{1}{3}|Z|^{2} \delta N_{X}^{2}, \sigma^{2} M_{Y}=\sigma^{2} Y+\frac{1}{3}|Z|^{2} \delta N_{Y}^{2}$, the quantities $\delta N_{X}, \delta N_{Y}$ being the Hough bin size along $N_{X}$, $N_{Y}$. The intersection bins, i.e. the track candidates, are then ordered according to their number of clusters and according to their $\chi^{2}$ likelihood. These candidates are accepted with first preferring the longer and bigger likelihood ones, with a subsequent removal of their clusters from the Hough table, thus can be regarded as a maximum likelihood track finding method. The cost is merely linear in number of clusters $\times$ number of Hough plane-band bins of a typical cluster. The Hough table is implemented using a container not storing the bins unoccupied by clusters, and thus reducing the memory requirement to approximately the square-root of the total Hough binning.

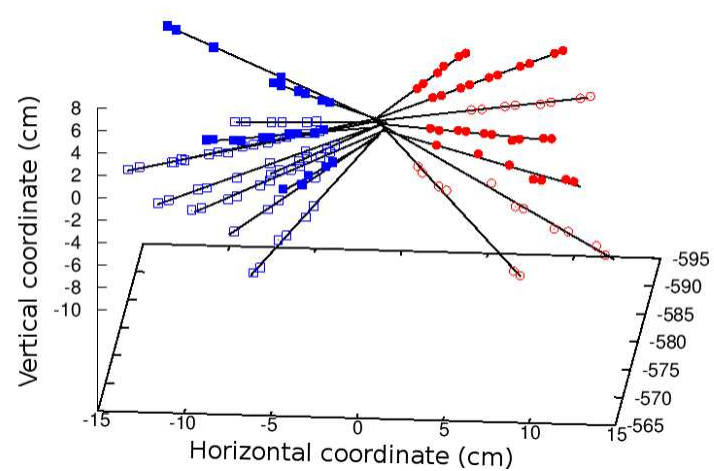

Figure 14: Track finding for different number of measured clusters (10 superimposed events, points: clusters, lines: tracks).

The cluster and reconstruction, calibration and analysis software is implemented in the standard offline software framework, Shine, of the NA61 experiment [29]. The performance of the event reconstruction was verified by eye scans over sample of 500 events, and proved to be close to ideal. Figure 14 shows track reconstruction in operation for tracks with different number of measured clusters.

After track reconstruction the fitted track may be extrapolated to the constant $Z$ plane intersecting with the target. Figure 15] shows the distributions of these extrapolated intersection point coordinates for the target in and the target out data samples. The contribution of interactions within the target is clearly visible. The contribution from non-target tracks in the target region is well below the percent level, demonstrating the success of background suppression. 

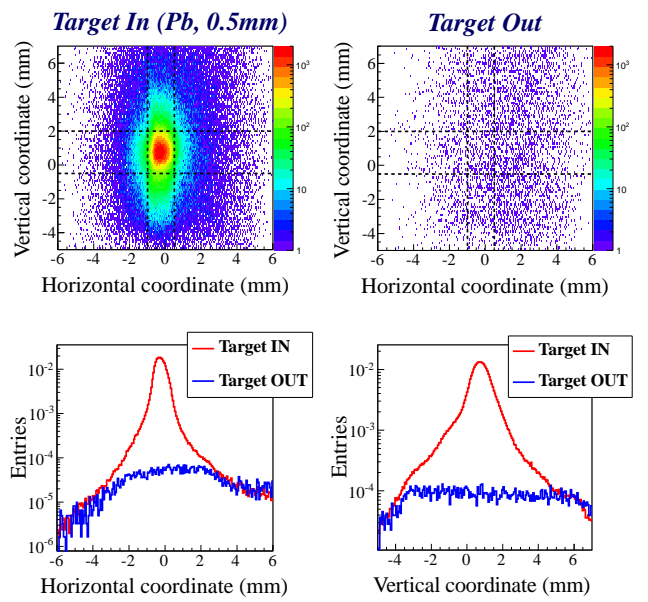

Figure 15: Distributions of the extrapolated track points to the target plane (upper left: target in, upper right: target out). Contribution of the target plane is clearly visible. Lower panels show the horizontal and vertical distributions, for the regions indicated by lines on the upper left panel.

\subsection{Ionization for a given range: demonstration of $Z=1$ particle identification}

The particle identification concept adapted for the LMPD is the simultaneous range and ionization $(\mathrm{dE} / \mathrm{dx})$ measurement. The former is a direct result of a reliable tracking algorithm, whereas the latter requires precise calibration taking into account angular effects as well. However, already on the level of reconstructed data, the demonstration of the concept is possible. Figure 16 top left panel shows those tracks which have stopped in the second absorber, that is, measured in the first two detection layers (4 pad rows) without continuation in the sensitive volume. The ionization added up on the first two pad rows (first detection layer) correlates well with the ionization on the second pair of pad rows (second detection layer), and a marked peak around $60 \mathrm{keV}$ matches well with the expected most probable ionization for protons (see Table 2), but it contains also the deuterons. A peak at four times larger ionization corresponds to alphas and ${ }^{3} \mathrm{He}$.

The other panels of Figure 16 shows the similar 2 dimensional energy deposition distribution for the tracks stopped in the given absorber. The $\mathrm{Z}=1$ and $\mathrm{Z}=2$ peaks are visible on all plots.

\subsection{Comparison to PAI simulation of $d E / d x$}

The measured $\mathrm{dE} / \mathrm{dx}$ distributions in the first detection layer for angles which are closely perpendicular to the absorbers may be compared to a simulation based on
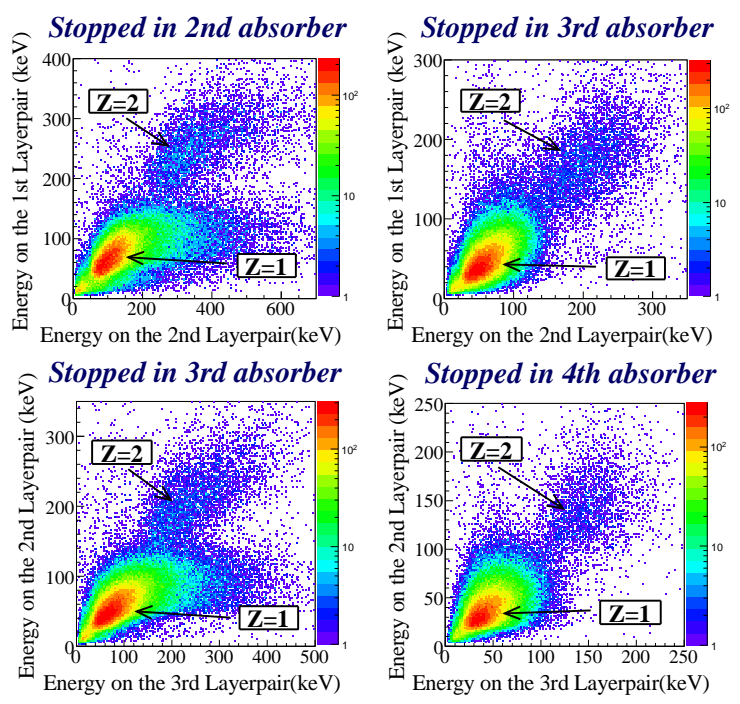

Figure 16: Correlation of deposited energy (dE/dx) for stopped particles in adjacent layers. Peaks for $\mathrm{Z}=1$ (mainly protons, but also deuterons) and $\mathrm{Z}=2(\mathrm{He})$ are clearly visible.

the PAI model [17], calculated by the authors. On the left panel of Figure 17, the simulation result is shown, for proton tracks which are stopped in any of the four absorbers. The right panel shows the actual measurement in the LMPD in a physics run. The differences are due to the fact that the simulation includes only protons, whereas in the measured data protons, deuterons and pions are also pesented. Though this figure serves only for the purpose of a qualitative comparison and needs refinements from both the simulation and the data analysis sides, the similarity is clear, and proves the validity of the proposed PID concept based on $\mathrm{dE} / \mathrm{dx}$ and range measurement.
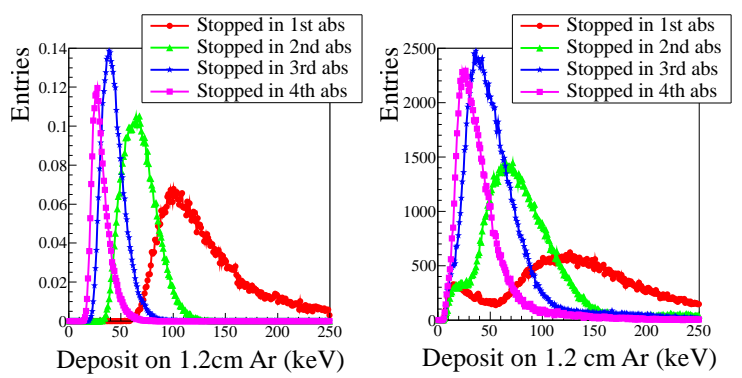

Figure 17: Distribution of ionization $(\mathrm{dE} / \mathrm{dx})$ for the first measured layer: comparison of simulation based on the PAI model (left panel) and the measurement (right panel). 


\section{Conclusions}

The paper has presented the design, construction and operation of the Low Momentum Particle Detector, a new component of the CERN NA61 Experiment. It has been demonstrated that with this small TPC a highly reliable tracking is possible for tracks emitted from the target, and high ionization tracks can be tagged as gray particle candidates. In some momentum ranges, defined by absorbers, particle identification is directly possible, differentiating $\mathrm{Z}=1$ particles from pions or heavy fragments. The detector will provide useful input for understanding slow particle production in hadron-nucleus interactions, correlating production properties with the production of forward particles, and especially clarifying the role of "black" and "gray" protons in collision centrality determination.

\section{Acknowledgements}

The authors acknowledge the financial support from the Hungarian National Research Fund (OTKA) 68506 and the Momentum ("Lendület") Programme of the Hungarian Academy of Sciences. We wish to thank for the support of the "REGaRD" group of the Wigner RCP in Budapest, and the technical help from M. Wensween and all members of the CERN NA61/Shine Collaboration.

\section{References}

[1] J. Benecke, T. T. Chou, C. N. Yang, E. Yen, Phys. Rev. 188, 2159 (1969)

[2] Heckman et al., Phys. Rev. C17, 1651 (1978)

[3] S. Fredriksson, G. Eilam, G. Berlad and L. Bergstrom, Phys. Rept. 144, 187 (1987)

[4] G. Barr et al. Eur.Phys.J. C49 919-945 (2007)

[5] N. Abgrall et al. CDS:CERN-SPSC-2006-034

[6] N. Abgrall et al. (NA61/SHINE Collaboration), CERN-PH-EP2014-003.

[7] F. Sikler, hep-ph/0304065

[8] K. Braune et al., Zeit. Phys. C13, 191 (1982)

[9] M. L. Cherry et al., Phys. Rev. D50, 4272 (1994)

[10] O. Chvala et al. Eur.Phys.J. C73, 2329 (2013)

[11] Eur.Phys.J. C73, 2364 (2013)

[12] P. A. Piroue, A. J. S. Smith, Phys. Rev. 148, 1315 (1966)

[13] H. H. Gutbrod et al., Phys. Rev. Lett. 37, 667 (1976)

[14] W. M. Yeager et al., Phys. Rev. D16, 1294 (1977)

[15] A. Abduzhamilov et al. (BATON ROUGE-KRAKOWMOSCOW-TASHKENT), Phys. Rev. D39, 86 (1989)

[16] A. Dabrowska et al. (KLM), Phys. Rev. D47, 1751 (1993)

[17] W. W. M. Allison, J. H. Cobb, Ann. Rev. Nucl. Part. Sci. 30, 253 (1980)

[18] S. Afanasiev et al. (the NA49 Collaboration), Nucl. Instrum. Meth. A430 (2-3), 210 (1999)

[19] http://garfield.web.cern.ch/garfield/
[20] N. Majumdar, S. Mukhopadhyay, Journal of Instrumentation 2 P09006 (2007)

[21] D. Varga, G. Kiss, G. Hamar, Gy. Bencedi, Nucl. Instr. Meth. A698, 11 (2013)

[22] S. A. Kleinfelder, IEEE Trans. on Nucl. Sci. 37, 1230 (1990)

[23] F. Bieser et al., Nucl. Instr. Meth. A385, 535 (1997)

[24] G. Rubin et al., Proceedings of the Fifth Workshop on Electronics for LHC Experiments, p.493 (1999)

[25] W. Carena et al., Proceedings of the 10th Workshop on Electronics for LHC Experiments, p.273 (2004)

[26] B. Lasiuk, C. A. Whitten, STAR Note 360 (1998)

[27] D. Carbone et al., Eur. Phys. J. A48, 60 (2012)

[28] P. V. C. Hough, Proc. Int. Conf. High Energy Accelerators and Instrumentation, 554 (1959)

[29] R. Sipos et al., J. Physics Conf. Ser 396, 022045 (2012) 